\title{
On equivalent representations and properties of faces of the cone of copositive matrices
}

\author{
Kostyukova O.I.* $\quad$ Tchemisova T.V. ${ }^{\dagger}$
}

December 7, 2020

\begin{abstract}
The paper is devoted to a study of the cone $\mathcal{C O}^{p}$ of copositive matrices. Based on the known from semi-infinite optimization concept of immobile indices, we define zero and minimal zero vectors of a subset of the cone $\mathcal{C O P}^{p}$ and use them to obtain different representations of faces of $\mathcal{C O P}^{p}$ and the corresponding dual cones. We describe the minimal face of $\mathcal{C O} \mathcal{P}^{p}$ containing a given convex subset of this cone and prove some propositions that allow to obtain equivalent descriptions of the feasible sets of a copositive problems and may be useful for creating new numerical methods based on their regularization.
\end{abstract}

Key words. Cone of copositive matrices; copositive programming; face; zero vectors of a subset of copositive matrices; regularization; face reduction; the Slater condition.

AMS subject classification. 49N15, 90C25, 90C34, 90C46

\section{Introduction}

This work is motivated by our main challenge: study of Copositive Programming (CoP) problems and their properties. CoP deals with a special class of conic problems and can be considered as an optimization over the convex cone of so-called copositive matrices (i.e. matrices which are positive semidefinite on the non-negative orthant). Copositive problems attract the attention of researchers as they have many interesting uses (see e.g. $[1,5,6]$, and the references therein). It should be noted here that $\mathrm{CoP}$ can be seen as a generalization of Semidefinite Programming (SDP) and a particular case of Semi-infinite Programming (SIP), whose important applications are well known [2, 27, 28].

An optimization problem is called regular if its constraints satisfy some additional (regularity) conditions, so-called constraint qualifications (CQs). The regularity of an optimization problem guarantees that the first-order necessary optimality conditions can be formulated in the form of so-called Karush-Kuhn-Tucker (KKT) system [18, 19] and

*Institute of Mathematics, National Academy of Sciences of Belarus, Surganov str. 11, 220072, Minsk, Belarus (kostyukova@im. bas-net.by).

†Mathematical Department, University of Aveiro, Campus Universitario Santiago, 3810-193, Aveiro, Portugal (tatiana@ua.pt). 
the strong duality relation is satisfied [14]. In most of the works on CoP and SIP, the study is conducted on the assumption that some regularity conditions are satisfied (see e.g. [1] for linear CoP and [12, 13, 20] for convex SIP).

In the cases where regularity conditions are not met, there is no guarantee that an optimal solution satisfies the KKT type optimality conditions, the first-order optimality conditions of the Fritz John type (see Theorem 2 in [19]) become degenerate (they are satisfied for all feasible solutions and hence are not informative), and the strong duality relation may fail. This creates difficulties in numerical solving problems. To overcome these obstacles, special regularization procedures can be applied, allowing to rewrite the original problem in an equivalent form for which certain regularity conditions are met. This approach is based on the fact that as a rule, the violation of CQs is caused by an unhappy description of the feasible set of optimization problem. To obtain non-degenerate optimality conditions, one needs a "good" representation of the feasible set.

In conic optimization, the regularization procedures are based on the so-called facial reduction algorithms (FRAs). To perform a constructive regularization procedure for a conic problem, it is necessary to explicitly describe certain faces of its feasible cone (the faces containing a given convex subset of this cone) and their dual ones. For SDP problems, which are a particular case of conic problems, the properties of faces of the cone of semidefinite matrices are well studied. This allowed to describe constructive regularization procedures for SDP and develop a duality theory satisfying strong duality conditions without regularity assumption. In particular, in [23, 24], etc., a dual problem for SDP is explicitly formulated in the form of an Extended Lagrange Dual Problem (ELDP). Several attempts have been done to obtain regularization procedures for general conic problems (see e.g. [7, 26]), but the procedures described there are implicit and do not permit to obtain explicit strong dual formulations. There is no such duality theory for CoP as well. This can be explained by the fact that the structure and properties of faces of the cone of copositive matrices and the respective duals are not well studied yet [4]. It worth mentioning that several attempts have been done to study the facial structure of the cones of copositive and completely positive matrices. Thus in $[3,9,11]$, the authors give explicit characterizations of extreme rays (faces of dimension one) of copositive cones of dimensions five and six. In $[8,9]$, some properties of special types of faces (minimal and maximal ones) are studied. Nevertheless, until now, for the cone of copositive matrices, faces of this cone and the corresponding dual cones are not well studied.

The aims of the paper are as follows:

- for a given convex subset of the set of copositive matrices, to define the corresponding set of all its zeros and minimal zeroes and to study some of their properties;

- given a face of the cone of copositive matrices, to obtain its representation in terms of the corresponding minimal zeros and describe explicitly the dual cone to this face;

- for a given closed convex subset of the cone of copositive matrices, to derive an explicit description of the cone's minimal face containing this subset in terms of the corresponding set of minimal zeros;

- to obtain equivalent "good" descriptions of the feasible sets of convex CoP problems.

The rest of the paper is structured as follows. In section 2, we collect notation, basic definitions, and prove some important results which will be used in this paper. In section 3 , for a convex closed subset of the cone of copositive matrices, we define its minimally active element and prove the existence of such an element. Section 4 contains three 
equivalent representations of faces of the copositive cone, and in section 5, we deduce alternative representations of the dual cones to the faces. In section 6 , we describe the minimal face of the cone of copositive matrices containing a given convex set and prove some corollaries that may be useful for creating new numerical methods based on the minimal cone representations and regularization procedures for copositive problems. In section 7 , we consider equivalent descriptions of the feasible set of a copositive problem. The final section 8 contains some conclusions.

\section{Notation, basic definitions, and important prelim- inary results}

Given an integer $p>1$, consider the vector space $\mathbb{R}^{p}$ with the standard orthogonal basis $\left\{e_{k}, k=1,2, \ldots, p\right\}$. Denote by $\mathbb{R}_{+}^{p}$ the set of all $p$ - vectors with non-negative components, by $\mathcal{S}(p)$ and $\mathcal{S}_{+}(p)$ the space of real symmetric $p \times p$ matrices and the cone of symmetric positive semidefinite $p \times p$ matrices, respectively, and let $\mathcal{C O} \mathcal{P}^{p}$ stay for the cone of symmetric copositive $p \times p$ matrices:

$$
\mathcal{C O} \mathcal{P}^{p}:=\left\{D \in \mathcal{S}(p): t^{\top} D t \geq 0 \forall t \in \mathbb{R}_{+}^{p}\right\}
$$

The space $\mathcal{S}(p)$ is considered here as a vector space with the trace inner product $A \bullet B:=$ trace $(A B)$. Denote

$$
T:=\left\{t \in \mathbb{R}_{+}^{p}: \mathbf{e}^{\top} t=1\right\}
$$

with $\mathbf{e}=(1,1, \ldots, 1)^{\top} \in \mathbb{R}^{p}$. It is evident that the cone $\mathcal{C O} \mathcal{P}^{p}$ can be equivalently described as follows: $\mathcal{C O} \mathcal{P}^{p}=\left\{D \in \mathcal{S}(p): t^{\top} D t \geq 0 \forall t \in T\right\}$.

Given a vector $t=\left(t_{k}, k \in P\right)^{\top} \in \mathbb{R}_{+}^{p}$ with $P:=\{1,2, \ldots, p\}$, introduce the sets

$$
P_{+}(t):=\left\{k \in P: t_{k}>0\right\} \text { and } P_{0}(t):=P \backslash P_{+}(t)
$$

Given a set $\mathcal{B} \subset \mathbb{R}^{p}$ denote by conv $\mathcal{B}$ the convex hull of $\mathcal{B}$, by int $\mathcal{B}$ the interior, by relint $\mathcal{B}$ the relative interior and by $\operatorname{cl} \mathcal{B}$ the closure of this set. For the set $\mathcal{B}$ and a point $l=\left(l_{k}, k \in P\right)$, denote by $\rho(l, \mathcal{B})$ the distance between this set and the point, $\rho(l, \mathcal{B}):=\min _{\tau \in \mathcal{B}} \sum_{k \in P}\left|l_{k}-\tau_{k}\right|$.

In our previous papers (see e.g. [15, 16, 17], and the references therein), considering convex SIP problems, for a constraint function satisfying the inequalities $f(x, t) \geq 0$ $\forall x \in X_{f}, \forall t \in \Upsilon$, where $X_{f} \subset \mathbb{R}^{n}$ is the convex set of feasible solutions, $\Upsilon \subset \mathbb{R}^{p}$ is a compact index set, we introduced the concept of immobile indices of the constraints and showed that this concept plays an important role in the study of optimality of solutions to these problems and regularity of their feasible sets. The set of immobile indices was defined as follows:

$$
T_{\text {im }}:=\left\{t \in \Upsilon: f(x, t)=0 \forall x \in X_{f}\right\} .
$$

In this paper, in a similar way we introduce a notion of zeros of convex subsets of the cone $\mathcal{C O P}^{p}$ and use them to describe faces of this cone, the dual cones corresponding to faces of $\mathcal{C O} \mathcal{P}^{p}$, and the minimal face containing a given convex subset.

For a matrix set $Q \subset \mathcal{C O} \mathcal{P}^{p}$ we will say that vector $t \in T$ is a zero of the set $Q$ if $t^{\top} D t=0 \forall D \in Q$. 
Denote by $T_{0}(Q)$ the set of all zeros of the given matrix set $Q$ :

$$
T_{0}(Q):=\left\{t \in T: t^{\top} D t=0 \quad \forall D \in Q\right\} .
$$

Note that if we consider a function $\varphi(D, t):=t^{\top} D t$ defined for $D \in \mathcal{S}(p), t \in T$, and satisfying the following conditions:

$$
\varphi(D, t) \geq 0 \forall D \in Q, \forall t \in T,
$$

where $Q \subset \mathcal{C O P} \mathcal{P}^{p}$, then $t \in T$ is an immobile index of this function if $t^{\top} D t=0 \forall D \in Q$. Therefore, the set $T_{0}(Q)$ of all zeros of the matrix set $Q$ is nothing but the set of immobile indices of the defined above function $\varphi$.

For a given subset $Q \subset \mathcal{C O} \mathcal{P}^{p}$, a vector $\tau \in T_{0}(Q)$ is called a minimal zero of $Q$ if there does not exist $t \in T_{0}(Q), t \neq \tau$, such that $P_{+}(t) \subset P_{+}(\tau)$.

Note that the introduced here definitions of zeros and minimal zeros of a given set $Q \subset \mathcal{C O} \mathcal{P}^{p}$, generalize the concepts of zeros and minimal zeros introduced in [9] for a given matrix $A \in \mathcal{C O} \mathcal{P}^{p}$.

For the considered above set $Q$, let us say that it satisfies the Slater condition if

$$
\exists \bar{D} \in Q \text { such that } \bar{D} \in \operatorname{int} \mathcal{C O} \mathcal{P}^{p}=\left\{D \in \mathcal{S}(p): t^{\top} D t>0 \forall t \in T\right\} .
$$

Repeating the chain of proofs of Lemma 1 and Proposition 1 in [16], we can prove the following lemma.

Lemma 1 Given a closed convex subset $Q \subset \mathcal{C O P}^{p}$,

(i) the Slater condition (3) is equivalent to the emptiness of the set $T_{0}(Q)$;

(ii) the set $T_{0}(Q)$ of all zeros of the matrix set $Q$ is either empty or can be represented as a union of a finite number of convex closed bounded polyhedra.

It follows from Lemma 1 that if $T_{0}(Q) \neq \emptyset$, then the set conv $T_{0}(Q)$ is a convex bounded polyhedron with a finite number of vertices. If $T_{0}(Q)=\emptyset$, then, evidently, $\operatorname{conv} T_{0}(Q)=\emptyset$. Denote by

$$
Z_{Q}:=\left\{\tau(j), j \in J_{Q}\right\}, 0 \leq\left|J_{Q}\right|<\infty,
$$

the set of all vertices of the set conv $T_{0}(Q)$. Notice that $J_{Q}:=\emptyset$ when $T_{0}(Q)=\emptyset$.

From the definition of the set $T_{0}(Q)$, we conclude that for each matrix $D \in Q$, the elements $\tau(j), i \in J_{Q}$, of the set $Z_{Q}$ are optimal solutions of the problem

$$
\min t^{\top} D t \text { s.t. } t \in T \text {. }
$$

Hence

$$
e_{k}^{\top} D \tau(j)=0 \forall k \in P_{+}(\tau(j)) ; e_{k}^{\top} D \tau(j) \geq 0 \forall k \in P_{0}(\tau(j)), \forall j \in J_{Q}, \forall D \in Q .
$$

Define the sets

$$
M_{Q}(j):=\left\{k \in P: e_{k}^{\top} D \tau(j)=0 \forall D \in Q\right\}, j \in J_{Q} .
$$

It follows from $(6)$ that $P_{+}(\tau(j)) \subset M_{Q}(j) \forall j \in J_{Q}$.

Note that in this paper we consider different types of subsets $Q$ of the cone $\mathcal{C O} \mathcal{P}^{p}$. Therefore we include the reference on $Q$ in the designation of the sets (2), (4), and (7). 
Lemma 2 For a given convex closed set $Q \subset \mathcal{C O P}{ }^{p}$, the set of all its minimal zeros is either empty or finite and coincident with the set $\left\{\tau(j), j \in J_{Q}\right\}$ of all vertices of the set $\operatorname{conv} T_{0}(Q)$.

Proof. It is evident that if the set $T_{0}(Q)$ is empty, then the set of minimal zeros is empty too.

Suppose that $T_{0}(Q) \neq \emptyset$. Notice that since $Q \subset \mathcal{C O} \mathcal{P}^{p}$ and $\tau(j) \in T_{0}(Q)$ for all $j \in J_{Q}$, the relations (6) hold true.

First, let us show that all vertices of the set $\operatorname{conv} T_{0}(Q)$ are minimal zeros of the set $Q$. Suppose that, on the contrary, for some $j_{0} \in J_{Q}$, the corresponding zero $\tau\left(j_{0}\right)$ is not minimal for the set $Q$. Then there exists another zero $\bar{t} \in T_{0}(Q)$ such that $P_{+}(\bar{t}) \subset$ $P_{+}\left(\tau\left(j_{0}\right)\right), \bar{t} \neq \tau\left(j_{0}\right)$. Consequently, for a sufficiently small $\theta>0$, we have

$$
\bar{\tau}:=\left(\tau\left(j_{0}\right)-\theta \bar{t}\right) /(1-\theta) \in T, \quad \bar{\tau} \neq \bar{t} .
$$

Let us show that $\bar{t}^{\top} D \tau\left(j_{0}\right)=0$ for all $D \in Q$. In fact, taking into account (6), we get

$$
\bar{t}^{\top} D \tau\left(j_{0}\right)=\sum_{k \in P} \bar{t}_{k} e_{k}^{\top} D \tau\left(j_{0}\right)=\sum_{k \in P_{+}\left(\tau\left(j_{0}\right)\right)} \bar{t}_{k} e_{k}^{\top} D \tau\left(j_{0}\right)=0 \quad \forall D \in Q
$$

For any $D \in Q$, from (8) and the equalities $\bar{t}^{\top} D \bar{t}=\left(\tau\left(j_{0}\right)\right)^{\top} D \tau\left(j_{0}\right)=\bar{t}^{\top} D \tau\left(j_{0}\right)=0$ it follows that $\bar{\tau}^{\top} D \bar{\tau}=0$. Hence $\bar{\tau} \in T_{0}(Q)$. As a result, we obtain

$$
\tau\left(j_{0}\right)=(1-\theta) \bar{\tau}+\theta \bar{t}, \theta \in(0,1), \bar{\tau} \in \operatorname{conv} T_{0}(Q), \bar{t} \in \operatorname{conv} T_{0}(Q), \bar{t} \neq \bar{\tau}
$$

But these relations contradict the assumption that $\tau\left(j_{0}\right)$ is a vertex of the set $\operatorname{conv} T_{0}(Q)$. Thus, it is proved that all vertices $\tau(j), j \in J_{Q}$, are minimal zeros of the set $Q$.

To prove that there isn't a single minimal zero outside the set $\left\{\tau(j), j \in J_{Q}\right\}$, c onsider a zero $t^{*} \in T_{0}(Q) \backslash\left\{\tau(j), j \in J_{Q}\right\} \subset \operatorname{conv} T_{0}(Q)$. By construction, it admits the following representation:

$$
t^{*}=\sum_{j \in J^{*}} \alpha_{j} \tau(j), \sum_{j \in J^{*}} \alpha_{j}=1, \alpha_{j}>0 \forall j \in J^{*} \subset J_{Q},\left|J^{*}\right| \geq 2 .
$$

It follows from this representation that $P_{+}\left(t^{*}\right)=\bigcup_{j \in J^{*}} P_{+}(\tau(j))$, which implies

$$
P_{+}(\tau(j)) \subset P_{+}\left(t^{*}\right), \tau(j) \neq t^{*}, \quad \tau(j) \in T_{0}(Q) \forall j \in J^{*} .
$$

By definition, the obtained inclusions mean that $t^{*}$ is not a minimal zero of the set $Q$. The proposition is proved.

In what follows, the set $Z_{Q}$ will be also called the set of minimal zeros of the set $Q$.

Consider the set $T$ defined in (1). For a given nonempty finite subset

$$
V:=\{t(i) \in T, i \in I\}, 0<|I|<\infty
$$

define the number

$$
\sigma(V):=\min \left\{t_{k}(i), k \in P_{+}(t(i)), i \in I\right\} .
$$

By definition, evidently, $\sigma(V)>0$. 
Introduce the following sets:

$$
\begin{gathered}
\Omega(V):=\{t \in T: \rho(t, \operatorname{conv} V) \geq \sigma(V)\} \\
\mathcal{N}(V):=\{t \in T: \rho(t, \operatorname{conv} V) \leq \sigma(V)\} .
\end{gathered}
$$

Note that, by construction, $T=\Omega(V) \cup \mathcal{N}(V)$ and $\Omega(V) \cap \mathcal{N}(V)=\{t \in T: \rho(t, \operatorname{conv} V)=$ $\sigma(V)\}$.

In the rest of this section, we will prove two auxiliary statements that will be used to justify the main results of this paper.

Proposition 1 Let the set $V$ be given in (9), and the corresponding number $\sigma(V)$ and set $\mathcal{N}(V)$ defined in (10) and (12). Then for any $t \in \mathcal{N}(V)$, there exists $t\left(i_{0}\right) \in V$ such that $P_{0}(t) \subset P_{0}\left(t\left(i_{0}\right)\right)$.

Proof. Consider $t \in \mathcal{N}(V)$. By construction, there exists $\tau \in \operatorname{conv} V$ and a nonempty set $I_{*} \subset I$ such that

$$
\rho(t, \operatorname{conv} V)=\rho(t, \tau) \leq \sigma(V) ; \tau=\sum_{i \in I_{*}} \alpha_{i} t(i), \sum_{i \in I_{*}} \alpha_{i}=1, \alpha_{i}>0 \forall i \in I_{*} .
$$

Suppose that $P_{0}(t) \not \subset P_{0}(t(i)) \forall i \in I$. It is evident that these relations are equivalent to the following ones: $P_{0}(t) \cap P_{+}(t(i)) \neq \emptyset \quad \forall i \in I$. Taking into account the latest inequalities and (13), we get

$$
\begin{aligned}
\sigma(V) & \geq \rho(t, \tau)=\sum_{k \in P}\left|t_{k}-\tau_{k}\right|=\sum_{k \in P_{+}(t)}\left|t_{k}-\tau_{k}\right|+\sum_{k \in P_{0}(t)} \tau_{k} \\
& \geq \sum_{k \in P_{0}(t)} \tau_{k}=\sum_{k \in P_{0}(t)} \sum_{i \in I_{*}} \alpha_{i} t_{k}(i)=\sum_{i \in I_{*}} \alpha_{i} \sum_{k \in P_{0}(t) \cap P_{+}(t(i))} t_{k}(i) \geq \sigma(V) .
\end{aligned}
$$

Thence, $\rho(t, \tau)=\sigma(V)$ and in the chain of inequalities above, we can replace the inequality symbol by the equality one. Then $\sum_{k \in P_{+}(t)}\left|t_{k}-\tau_{k}\right|=0$. Hence $t_{k}=\tau_{k} \forall k \in P_{+}(t)$. From the latter equalities and the following relations:

$$
t \geq 0, \tau \geq 0, \mathbf{e}^{\top} t=\mathbf{e}^{\top} \tau=1 ; t_{k}=0 \quad \forall k \in P_{0}(t)
$$

we get the equality $t=\tau$. Then, taking into account relations (13), we conclude that $P_{0}(t)=\bigcap_{i \in I_{*}} P_{0}(t(i))$, which implies $P_{0}(t) \subset P_{0}(t(i)) \forall i \in I_{*}$.

Hence we obtain a contradiction with the assumption $P_{0}(t) \not \subset P_{0}(t(i)) \forall i \in I$. The proposition is proved.

Theorem 1 Consider the defined in (1) set $T$, any its subset $V$ in the form (9), and the corresponding set $\Omega(V)$ defined in (11). For any matrix $D \in S(p)$, the relations

$$
t^{\top} D t \geq 0 \forall t \in \Omega(V) \text { and } D t(i) \geq 0 \forall i \in I,
$$

imply

$$
t^{\top} D t \geq 0 \forall t \in T .
$$


Proof. Given a matrix $D \in S(p)$ and the set $V$ defined in (9), first notice that the relations $D t(i) \geq 0 \forall i \in I$, imply $t^{\top} D t \geq 0 \forall t \in \operatorname{conv}\{t(i), i \in I\}=\operatorname{conv} V$.

Suppose that for a given $D \in S(p)$, relations (14) hold true, but some of the inequalities in (15) are violated. Hence for a vector $\bar{t}$ given by

$$
\bar{t}:=\arg \left\{\min t^{\top} D t, \text { s.t. } t \in T\right\},
$$

we have $\bar{t}^{\top} D \bar{t}<0$ and $\bar{t} \in \mathcal{N}(V) \backslash \operatorname{conv} V$, where the set $\mathcal{N}(V)$ is defined in (10), (12). Since $\bar{t} \in \mathcal{N}(V)$, from Proposition 1 it follows:

$$
\exists i_{0} \in I \text { such that } P_{0}(\bar{t}) \subset P_{0}\left(t\left(i_{0}\right)\right) .
$$

Let us set $l:=\bar{t}-t\left(i_{0}\right)$. The vector $l$ is a feasible direction for $t\left(i_{0}\right)$ in the set $T$ as, evidently, $t\left(i_{0}\right)+\lambda\left(\bar{t}-t\left(i_{0}\right)\right) \in T$ for all $\lambda \in[0,1]$. It follows from (17) that $l_{k}=0 \quad \forall k \in P_{0}(\bar{t})$. Hence, the vector $l$ is a feasible direction for $\bar{t}$ in $T$ as well. As $l$ is a feasible direction for $t\left(i_{0}\right)$ and $\bar{t}$ in $T$, then there exists $\gamma_{0}>1$ such that for all $\gamma \in\left[0, \gamma_{0}\right]$, it holds: $t(\gamma):=t\left(i_{0}\right)+\gamma l=t\left(i_{0}\right)+\gamma\left(\bar{t}-t\left(i_{0}\right)\right) \in T$.

Define the function

$$
f(\gamma):=t^{\top}(\gamma) D t(\gamma)=\gamma^{2} a+2 \gamma b+c, \gamma \in\left[0, \gamma_{0}\right]
$$

where $a:=l^{\top} D l, b:=l^{\top} D t\left(i_{0}\right)$, and $c:=\left(t\left(i_{0}\right)\right)^{\top} D t\left(i_{0}\right)$. According to (16), we have

$$
\min _{0 \leq \gamma \leq \gamma_{0}} f(\gamma)=f\left(\gamma^{*}=1\right)=a+2 b+c
$$

Since $\gamma^{*}=1 \in\left(0, \gamma_{0}\right)$, then $0=\frac{d f\left(\gamma^{*}\right)}{d \gamma}=2 a+2 b$, which implies $a=-b$ and, equivalently, $l^{\top} D l=-l^{\top} D t\left(i_{0}\right)$. Then, taking into account the definition of vector $l$, we conclude that

$$
\bar{t}^{\top} D \bar{t}=\bar{t}^{\top} D t\left(i_{0}\right)
$$

Remind that, by assumption, it holds $\bar{t}^{\top} D \bar{t}<0$. On another hand, the inequalities $D t\left(i_{0}\right) \geq 0$ and $\bar{t} \geq 0$ imply $\bar{t} D t\left(i_{0}\right) \geq 0$. The obtained contradiction with the equality (18) completes the proof.

\section{$3 \quad$ Minimally active elements of a set $Q \subset \mathcal{C O} \mathcal{P}^{p}$}

Given a matrix $A \in \mathcal{C O} \mathcal{P}^{p}$, let $T_{0}(A)$ be the set of its zeros defined in (2) with $Q$ replaced by $A$. Denote by

$$
\left.Z_{A}:=\left\{\tau(j) \in T_{0}(A), j \in J_{A}\right)\right\},\left|J_{A}\right|<\infty,
$$

the set of all vertices (extremal points) of the set conv $T_{0}(A)$ which coincides with the set of minimal zeros of $A$ (see Lemma 2 ), and introduce the sets

$$
M_{A}(j):=\left\{k \in P: e_{k}^{\top} A \tau(j)=0\right\}, j \in J_{A} .
$$

Let $Q$ be a convex closed subset of the cone $\mathcal{C O} \mathcal{P}^{p}$.

Definition 1 A matrix $A \in Q$ is called minimally active element of the set $Q$ if for any $D \in Q$, it holds

$$
T_{0}(A) \subset T_{0}(D) \text { and } T_{0}(A)=T_{0}(D) \Longrightarrow M_{A}(j) \subset M_{D}(j) \forall j \in J_{A}=J_{D} .
$$


Note that from the definition, it follows that if $A \in Q$ and $T_{0}(A)=\emptyset$, then $A$ is a minimally active element of the set $Q$.

The main result of this section is the proof of a theorem which ensures the existence of a minimally active element of any convex closed subset of the cone $\mathcal{C O}^{p}$.

Given a convex closed matrix set $Q \subset \mathcal{C O} \mathcal{P}^{p}$, consider the sets $T_{0}(Q)$ and $Z_{Q}$ of all zeros and minimal zeros of $Q$, and the sets $M_{Q}(j), j \in J_{Q}$, defined in (2), (4), and (7).

Theorem 2 Given a convex closed set $Q \subset \mathcal{C O P}^{p}$, suppose that the set $T_{0}(Q)$ of all zeros of $Q$ is not empty. Then there exists a matrix $\bar{D} \in Q$ such that

$$
\begin{aligned}
& t^{\top} \bar{D} t>0 \forall t \in T \backslash T_{0}(Q) ; t^{\top} \bar{D} t=0 \forall t \in T_{0}(Q) ; \\
& e_{k}^{\top} \bar{D} \tau(j)>0 \forall k \in P \backslash M_{Q}(j), \forall j \in J_{Q} .
\end{aligned}
$$

Proof. Given a convex closed set $Q \subset \mathcal{C O}^{p}$, consider the set $Z_{Q}=\left\{\tau(j), j \in J_{Q}\right\}$ of all its minimal zeros. Denote

$$
\begin{aligned}
& U:=\left\{(i, j): i \in J_{Q}, j \in J_{Q}, i \neq j\right\} \\
& U_{+}:=\left\{(i, j) \in U: \exists D(i, j) \in Q,(\tau(i))^{\top} D(i, j) \tau(j)>0\right\} .
\end{aligned}
$$

By definitions (7) and (20), for any $j \in J_{Q}$ and any $k \in P \backslash M_{Q}(j)$ there exists a matrix $A(k, j) \in Q$ such that $e_{k}^{\top} A(k, j) \tau(j)>0$, and for any $(i, j) \in U_{+}$there exists a matrix $D(i, j) \in Q$ such that $(\tau(i))^{\top} D(i, j) \tau(j)>0$.

Consider a matrix

$$
\widetilde{D}:=\sum_{(i, j) \in U_{+}} \alpha_{i j} D(i, j)+\sum_{j \in J_{Q}} \sum_{k \in P \backslash M_{Q}(j)} \beta_{k j} A(k, j),
$$

where the coefficients $\alpha_{i j},(i, j) \in U_{+}$, and $\beta_{k j}, k \in P \backslash M_{Q}(j), j \in J_{Q}$, are such that

$\sum_{(i, j) \in U_{+}} \alpha_{i j}+\sum_{j \in J_{Q}} \sum_{k \in P \backslash M_{Q}(j)} \beta_{k j}=1, \alpha_{i j}>0 \forall(i, j) \in U_{+}, \beta_{k j}>0 \forall k \in P \backslash M_{Q}(j), \forall j \in J_{Q}$.

Notice that since the set $Q$ is convex, it holds $\widetilde{D} \in Q$. Let us show that the following inequalities are valid:

$$
\begin{gathered}
t^{\top} \widetilde{D} t>0 \forall t \in\left(\operatorname{conv} \mathrm{Z}_{\mathrm{Q}}\right) \backslash T_{0}(Q) ; \\
e_{k}^{\top} \widetilde{D} \tau(j)>0 \forall k \in P \backslash M_{Q}(j), \forall j \in J_{Q} .
\end{gathered}
$$

Inequalities (23) are valid by construction. Moreover, it holds (see (20))

$$
(\tau(i))^{\top} \widetilde{D} \tau(j)=0 \text { if }(i, j) \in U \backslash U_{+}, \quad(\tau(i))^{\top} \widetilde{D} \tau(j)>0 \text { if }(i, j) \in U_{+} .
$$

To prove (22), let us suppose that, on the contrary, there exists $\bar{t} \in\left(\right.$ conv $\left.\mathrm{Z}_{\mathrm{Q}}\right) \backslash T_{0}(Q)$ such that

$$
\bar{t}^{\top} \widetilde{D} \bar{t}=0 \text {. }
$$

From the condition $\bar{t} \in \operatorname{conv} \mathrm{Z}_{\mathrm{Q}}$, it follows that for some set $\bar{J} \subset J_{Q}$, it holds

$$
\bar{t}=\sum_{j \in \bar{J}} \alpha_{j} \tau(j), \text { where } \sum_{j \in \bar{J}} \alpha_{j}=1, \alpha_{j}>0 \forall j \in \bar{J} .
$$


Then we can rewrite (25) in the form

$$
0=\sum_{j \in \bar{J}}\left(\alpha_{j} \tau(j)\right)^{\top} \widetilde{D} \sum_{i \in \bar{J}} \alpha_{i} \tau(i)=\sum_{i \in \bar{J}} \sum_{j \in \bar{J}} \alpha_{i} \alpha_{j}(\tau(i))^{\top} \widetilde{D} \tau(j)
$$

wherefrom, taking into account (24), we obtain $(i, j) \in U \backslash U_{+} \forall i \in \bar{J}, \forall j \in \bar{J}, i \neq j$. Therefore $(\tau(i))^{\top} D \tau(j)=0 \forall i \in \bar{J}, \forall j \in \bar{J}, \forall D \in Q$. Hence

$$
\bar{t}^{\top} D \bar{t}=\sum_{i \in \bar{J}} \sum_{j \in \bar{J}} \alpha_{i} \alpha_{j}(\tau(i))^{\top} D \tau(j)=0 \quad \forall D \in Q,
$$

and $\bar{t} \in T_{0}(Q)$, which contradicts the condition $\bar{t} \in\left(\operatorname{conv} \mathrm{Z}_{\mathrm{Q}}\right) \backslash T_{0}(Q)$.

Following the proof of Lemma 3 in [16], it is easy to show that there exists $\bar{A} \in Q$, such that

$$
t^{\top} \bar{A} t>0 \forall t \in \Omega\left(Z_{Q}\right)
$$

where the set $\Omega\left(Z_{Q}\right)$ is defined in (9)-(11) with $V=Z_{Q}$.

Consider a matrix

$$
\bar{D}:=\frac{1}{2}(\widetilde{D}+\bar{A})
$$

where $\widetilde{D} \in Q$ is defined in (21) and $\bar{A} \in Q$ satisfies (26). By construction, $\bar{D} \in Q$ and it holds

$$
\begin{aligned}
& t^{\top} \bar{D} t>0 \forall t \in \Omega\left(Z_{Q}\right) \cup\left(\left(\operatorname{conv} \mathrm{Z}_{\mathrm{Q}}\right) \backslash T_{0}(Q)\right) \\
& e_{k}^{\top} \bar{D} \tau(j)>0 \forall k \in P \backslash M_{Q}(j), \forall j \in J_{Q} .
\end{aligned}
$$

To complete the proof of the theorem, let us show that for the matrix $\bar{D}$ defined above, the following inequalities hold:

$$
t^{\top} \bar{D} t>0 \quad \forall t \in\left\{t \in T: \rho\left(t, \operatorname{conv} Z_{Q}\right)<\sigma\left(Z_{Q}\right)\right\} \backslash \operatorname{convZ}_{\mathrm{Q}}
$$

Suppose that, on the contrary, there exists a vector $\bar{t}$,

$$
\bar{t} \in\left\{t \in T: \rho\left(t, \operatorname{conv} Z_{Q}\right)<\sigma\left(Z_{Q}\right)\right\} \backslash \operatorname{conv}_{Q},
$$

such that $\bar{t}^{\top} \bar{D} \bar{t}=0$. To come to a contradiction, we will take the next steps.

Step 0. Set $s:=1, t^{s}:=\bar{t} \in T$.

Step 1. For a given $t^{s} \in T$, if either $t^{s} \in \operatorname{conv}_{Q}$ or $\rho\left(t^{s}, \operatorname{conv} Z_{Q}\right) \geq \sigma\left(Z_{Q}\right)$ or $\left(t^{s}\right)^{\top} \bar{D} t^{s}>0$, then STOP. Otherwise, i.e. if

$$
t^{s} \in\left\{t \in T: \rho\left(t^{s}, \operatorname{conv} Z_{Q}\right)<\sigma\left(Z_{Q}\right)\right\} \backslash \operatorname{conv} \mathrm{Z}_{\mathrm{Q}} \text { and }\left(t^{s}\right)^{\top} \bar{D} t^{s}=0,
$$

go to the next step.

Step 2. As $\rho\left(t^{s}, \operatorname{conv} Z_{Q}\right)<\sigma\left(Z_{Q}\right)$, then, according to Proposition 1 , there exists an index $i_{s} \in J_{Q}$ such that $P_{0}\left(t^{s}\right) \subset P_{0}\left(\tau\left(i_{s}\right)\right)$, or equivalently, $P_{+}\left(\tau\left(i_{s}\right)\right) \subset P_{+}\left(t^{s}\right)$. Compute $\theta_{k}=\frac{t_{k}^{s}}{\tau_{k}\left(i_{s}\right)}>0 \forall k \in P_{+}\left(\tau\left(i_{s}\right)\right) \subset P_{+}\left(t^{s}\right)$, and set

$$
\theta:=\min \left\{\theta_{k}, k \in P_{+}\left(\tau\left(i_{s}\right)\right)\right\}>0 .
$$


Let us show that $\theta<1$. If suppose that $\theta \geq 1$, then, evidently,

$$
t_{k}^{s} \geq \tau_{k}\left(i_{s}\right) \forall k \in P_{+}\left(\tau\left(i_{s}\right)\right) .
$$

Taking into account the latter inequalities and the definition (1) of the set $T$, we get

$$
1=\sum_{k \in P_{+}\left(t^{s}\right)} t_{k}^{s} \geq \sum_{k \in P_{+}\left(\tau\left(i_{s}\right)\right)} t_{k}^{s} \geq \sum_{k \in P_{+}\left(\tau\left(i_{s}\right)\right)} \tau_{k}\left(i_{s}\right)=1 .
$$

From (30) and (31), it follows $t^{s}=\tau\left(i_{s}\right)$, which contradicts the assumption $t^{s} \notin$ conv $\mathrm{Z}_{\mathrm{Q}}$. Therefore, we have shown that $0<\theta<1$.

Set $t^{s+1}:=\left(t^{s}-\theta \tau\left(i_{s}\right)\right) /(1-\theta)$. Let us show that the following relations are satisfied:
a) $t^{s+1} \in T$;
b) $t^{s+1} \notin \operatorname{conv} Z_{Q}$;
c) $\left(t^{s+1}\right)^{\top} \bar{D} t^{s+1}=0$;
d) $\rho\left(t^{s+1}, \operatorname{conv} Z_{Q}\right)<\sigma\left(Z_{Q}\right)$.

The proof of these items is as follows.

a) By construction, $t^{s}-\theta \tau\left(i_{s}\right) \geq 0$. Hence $t^{s+1} \geq 0$ and $\mathbf{e}^{\top} t^{s+1}=1$. Therefore $t^{s+1} \in T$.

b) Suppose that, on the contrary, $t^{s+1} \in \operatorname{convZ}_{\mathrm{Q}}$. Then $t^{s}=(1-\theta) t^{s+1}+\theta \tau\left(i_{s}\right)$, where $\theta \in(0,1), \tau\left(i_{s}\right) \in \operatorname{convZ}_{\mathrm{Q}}$, and $t^{s+1} \in \operatorname{convZ}_{\mathrm{Q}}$. Hence $t^{s} \in \operatorname{conv}_{\mathrm{Q}}$ that contradicts (29). The obtained contradiction proves that $t^{s+1} \notin \operatorname{conv} Z_{\mathrm{Q}}$.

c) Since $t^{s+1} \in T$, then $\left(t^{s+1}\right)^{\top} \bar{D} t^{s+1} \geq 0$ and the following relations hold:

$$
\begin{aligned}
0 \leq\left(t^{s+1}\right)^{\top} \bar{D} t^{s+1} & =(1-\theta)^{-2}\left(t^{s}-\theta\left(\tau\left(i_{s}\right)\right)^{\top} \bar{D}\left(t^{s}-\theta\left(\tau\left(i_{s}\right)\right)\right)\right. \\
& =(1-\theta)^{-2}\left(-2\left(t^{s}\right)^{\top} \bar{D} \tau\left(i_{s}\right)\right) \leq 0 .
\end{aligned}
$$

Here we took into account that $t^{\top} D \tau(j) \geq 0 \quad \forall t \in T, \forall j \in J_{Q}, \forall D \in Q$.

From (32), it follows: $\left(t^{s+1}\right)^{\top} \bar{D} t^{s+1}=0$.

d) Suppose that $\rho\left(t^{s+1}, \operatorname{conv} Z_{Q}\right) \geq \sigma\left(Z_{Q}\right)$. From the condition a) and the inequalities (27), we get $\left(t^{s+1}\right)^{\top} \bar{D} t^{s+1}>0$, which contradicts c). Hence $\rho\left(t^{s+1}, \operatorname{conv} Z_{Q}\right)<$ $\sigma\left(Z_{Q}\right)$.

Notice that, by construction, the number of the null components of the vector $t^{s+1}$ is larger than that of the vector $t^{s}$ :

$$
\left|P_{0}\left(t^{s+1}\right)\right| \geq\left|P_{0}\left(t^{s}\right)\right|+1 .
$$

Let us substitute $s$ by $s+1$ and go to Step 1 .

On the Step 1, the situation STOP cannot happen since for any $s \geq 0$, by construction, relations (29) hold. On another hand, due to (33), one cannot repeat the described above procedure more than $p$ times. This contradiction proves that inequalities (28) hold true. The theorem is proved.

It follows from relations (19) that the constructed in the proof of Theorem 2 matrix $\bar{D}$ is a minimally active element of the set $Q$ in the case $T_{0}(Q) \neq \emptyset$. If $T_{0}(Q)=\emptyset$, then the set $Q$ satisfies the Slater condition, and, hence, there exists a matrix $\widehat{D} \in Q$ such that $T_{0}(\widehat{D})=\emptyset$. The matrix $\widehat{D}$ is a minimally active element in this case.

Corollary 1 Under the conditions of Theorem 2, there exists $\bar{D} \in Q$ such that

$$
t^{\top} \bar{D} t>0 \quad \forall t \in \Omega\left(Z_{Q}\right) ; e_{k}^{\top} \bar{D} \tau(j)>0 \quad \forall k \in P \backslash M_{Q}(j), \forall j \in J_{Q} .
$$




\section{On equivalent representations of a face of the cone $\mathcal{C O} \mathcal{P}^{p}$}

In this and the subsequent sections, based on the results above, we will obtain equivalent representations of faces of the cone $\mathcal{C O P}^{p}$ and their dual ones which will be useful for further research and some applications (for example, for regularization procedures and a study of the facial structure of $\left.\mathcal{C O} \mathcal{P}^{p}\right)$.

Given a finite non-empty vector set $V$ in the form (9), $V=\{t(i) \in T, i \in I\}$, and a set $\mathcal{L}:=\{L(i), i \in I\}$ of sets $L(i)$ such that $P_{+}(t(i)) \subset L(i) \subset P \forall i \in I$, consider a cone

$$
\mathcal{K}=\mathcal{K}(V, \mathcal{L}):=\left\{D \in \mathcal{C O} \mathcal{P}^{p}: e_{k}^{\top} D t(i)=0 \forall k \in L(i), \forall i \in I\right\} .
$$

It follows from the definition of the cone $\mathcal{K}$ that $(t(i))^{\top} D t(i)=0$ for all $i \in I$ and $D \in \mathcal{K}$. Consequently, for all $D \in \mathcal{K}$, the vectors $t(i), i \in I$, are optimal solutions of the problem (5). Hence

$$
e_{k}^{\top} D t(i)=0 \quad \forall k \in L(i) ; \quad e_{k}^{\top} D t(i) \geq 0 \quad \forall k \in P \backslash L(i), \forall i \in I, \forall D \in \mathcal{K},
$$

and the cone $\mathcal{K}$ defined in (35) can be rewritten in the form

$$
\begin{aligned}
\mathcal{K}=\widetilde{\mathcal{K}}:=\left\{D \in \mathcal{S}(p): t^{\top} D t \geq 0 \forall t \in T ;\right. \\
\left.\quad e_{k}^{\top} D t(i)=0 \forall k \in L(i), e_{k}^{\top} D t(i) \geq 0 \quad \forall k \in P \backslash L(i), \forall i \in I\right\},
\end{aligned}
$$

Proposition 2 The cone $\mathcal{K}$ defined in (35) is a face of $\mathcal{C O P}^{p}$.

Proof. Let

$$
A \in \mathcal{C O} \mathcal{P}^{p}, B \in \mathcal{C O} \mathcal{P}^{p} \text { such that }(A+B) \in \mathcal{K} .
$$

Remind that by definition, $\mathcal{K}$ is a face of $\mathcal{C O} \mathcal{P}^{p}$ if relations (38) imply $A \in \mathcal{K}$ and $B \in \mathcal{K}$. By construction, the condition $(A+B) \in \mathcal{K}$ is equivalent to the conditions

$$
(A+B) \in \mathcal{C O} \mathcal{P}^{p}, e_{k}^{\top}(A+B) t(i)=0 \quad \forall k \in L(i), \forall i \in I,
$$

which imply the equalities

$$
(t(i))^{\top}(A+B) t(i)=0,(t(i))^{\top} A t(i)=0,(t(i))^{\top} B t(i)=0 \forall i \in I .
$$

Moreover, from the conditions $A \in \mathcal{C O} \mathcal{P}^{p},(t(i))^{\top} A t(i)=0$, and $B \in \mathcal{C O} \mathcal{P}^{p},(t(i))^{\top} B t(i)=$ 0 , it follows that $A t(i) \geq 0$ and $B t(i) \geq 0 \quad \forall i \in I$. Taking into account these inequalities and the equalities in (39), we obtain

$$
e_{k}^{\top} A t(i)=0, e_{k}^{\top} B t(i)=0 \forall k \in L(i), \forall i \in I .
$$

By the definition of the cone $\mathcal{K}$, from the last equalities and relations (38), it follows that $A \in \mathcal{K}$ and $B \in \mathcal{K}$. The proposition is proved.

Remark 1 Note that if in (35) we have $L(i)=P_{+}(t(i)) \forall i \in I$, then the cone $\mathcal{K}$ is an exposed face of $\mathcal{C O P}^{p}$ (for the definition of the exposed face see e.g. [21]).

Remark 2 In what follows (see Corollary 2 in section 6), we will show that any face of the cone $\mathcal{C O P}^{p}$ can be presented in the form (35). 
Given the cone $\mathcal{K}$ defined in (35), consider the set $T_{0}(\mathcal{K})$ of all its zeros, the set $Z_{\mathcal{K}}=\left\{\tau(j), j \in J_{\mathcal{K}}\right\}$ of all vertices of the set $\operatorname{conv} T_{0}(\mathcal{K})$ (i.e. the set of minimal zeros of $\mathcal{K}$ ), and the sets $M_{\mathcal{K}}(j), j \in J_{\mathcal{K}}$, defined in (2), (4), and (7) with $Q=\mathcal{K}$. Evidently, for all $i \in I$, it holds $t(i) \in T_{0}(\mathcal{K})$.

Note that in general, there may exist a vector $\bar{t} \in T_{0}(\mathcal{K})$ such that $\bar{t} \notin \operatorname{conv}\{t(i), i \in$ $I\}$. In fact, suppose that $V=\{t(1), t(2)\}$ and $P_{+}(t(1)) \subset P_{+}(t(2))$. For a sufficiently small $\theta>0$, consider $t(\theta):=(t(2)-\theta t(1)) /(1-\theta)$. By construction, $t(\theta) \in T$ and $t(\theta) \notin \operatorname{conv}\{t(1), t(2)\}$. From the definitions of the sets $P_{+}(t), P_{0}(t)$, and the cone $\mathcal{K}$, and from the assumption $P_{+}(t(1)) \subset P_{+}(t(2))$, it follows :

$$
(t(i))^{\top} \operatorname{Dt}(i)=0, i=1,2 ; \quad(t(1))^{\top} \operatorname{Dt}(2)=0 \forall D \in \mathcal{K} .
$$

Then $(t(\theta))^{\top} D t(\theta)=0 \forall D \in \mathcal{K}$ and, hence, $t(\theta) \in T_{0}(\mathcal{K})$.

Lemma 3 The defined in (35) cone $\mathcal{K}$ coincides with the following cone $\overline{\mathcal{K}}$ :

$$
\begin{aligned}
\overline{\mathcal{K}}:=\left\{D \in \mathcal{S}(p): t^{\top} D t \geq 0 \forall t \in \Omega\left(Z_{\mathcal{K}}\right) ;\right. \\
\left.e_{k}^{\top} D \tau(j)=0 \forall k \in M_{\mathcal{K}}(j), e_{k}^{\top} D \tau(j) \geq 0 \forall k \in P \backslash M_{\mathcal{K}}(j), \forall j \in J_{\mathcal{K}}\right\},
\end{aligned}
$$

and there exists a matrix $\bar{D} \in \overline{\mathcal{K}}$ such that

$$
t^{\top} \bar{D} t>0 \quad \forall t \in \Omega\left(Z_{\mathcal{K}}\right), e_{k}^{\top} \bar{D} \tau(j)>0 \forall k \in P \backslash M_{\mathcal{K}}(j), \forall j \in J_{\mathcal{K}},
$$

where the sets $Z_{\mathcal{K}}=\left\{\tau(j), \in J_{\mathcal{K}}\right\}, M_{\mathcal{K}}(j), j \in J_{\mathcal{K}}$, and $\Omega\left(Z_{\mathcal{K}}\right)$ are defined in (4), (7) with $Q=\mathcal{K}$ and in (10),(11) with $V=Z_{\mathcal{K}}$.

Proof. First, let us show that the defined in (35) cone $\mathcal{K}$ coincides with the cone

$$
\begin{aligned}
\widehat{\mathcal{K}}:=\left\{D \in \mathcal{S}(p): t^{\top} D t \geq 0 \forall t \in T ;\right. \\
\left.\quad e_{k}^{\top} D \tau(j)=0 \forall k \in M_{\mathcal{K}}(j), e_{k}^{\top} D \tau(j) \geq 0 \forall k \in P \backslash M_{\mathcal{K}}(j), \forall j \in J_{\mathcal{K}}\right\} .
\end{aligned}
$$

From the definitions of the sets $\mathcal{K}$ and $T_{0}(\mathcal{K})$, it follows:

$$
\mathcal{K} \subset \mathcal{C O} \mathcal{P}^{p} \text { and }(\tau(j))^{\top} D \tau(j)=0 \forall j \in J_{\mathcal{K}}, \forall D \in \mathcal{K} .
$$

Then, for all $D \in \mathcal{K}$, the vectors $\tau(j), j \in J_{\mathcal{K}}$, are optimal solutions of problem (5). Hence relations (6) with $Q=\mathcal{K}$ hold true. Moreover, from (7) with $Q=\mathcal{K}$ it follows:

$$
e_{k}^{\top} D \tau(j)=0 \quad \forall k \in M_{\mathcal{K}}(j), \forall j \in J_{\mathcal{K}}, \forall D \in \mathcal{K} .
$$

These relations together with (6) imply the inclusions $\mathcal{K} \subset \widehat{\mathcal{K}}$ and $P_{+}(\tau(j)) \subset M_{\mathcal{K}}(j)$ $\forall j \in J_{\mathcal{K}}$.

Now, consider any vector $t(i) \in V$. Since, by construction, $t(i) \in T_{0}(\mathcal{K})$, then there exists a subset $J(i) \subset J_{\mathcal{K}}$ and numbers $\alpha_{j}=\alpha_{j}(i), j \in J(i)$, such that

$$
t(i)=\sum_{j \in J(i)} \alpha_{j} \tau(j), \sum_{j \in J(i)} \alpha_{j}=1, \alpha_{j}>0 \forall j \in J(i) .
$$

Hence, by construction,

$$
0=e_{k}^{\top} D t(i)=\sum_{j \in J(i)} \alpha_{j} e_{k}^{\top} D \tau(j) \forall k \in L(i), \forall D \in \mathcal{K} .
$$


Taking into account the equalities above and relations (6) (with $Q=\mathcal{K}$ ), we conclude that $e_{k}^{\top} D \tau(j)=0 \forall j \in J(i), \forall k \in L(i), \forall D \in \mathcal{K}$, wherefrom it comes

$$
L(i) \subset M_{\mathcal{K}}(j) \forall j \in J(i), \quad \forall i \in I .
$$

Now, we will show that $\widehat{\mathcal{K}} \subset \mathcal{K}$. Let $\bar{D} \in \widehat{\mathcal{K}}$ and $t(i) \in V$. It follows from (43) that

$$
e_{k}^{\top} \bar{D} t(i)=\sum_{j \in J(i)} \alpha_{j} e_{k}^{\top} \bar{D} \tau(j) \forall k \in P
$$

Taking into account these equalities and inclusions (44), we conclude that $e_{k}^{\top} \bar{D} t(i)=$ $0 \forall k \in L(i), \forall i \in I$, and hence, $\bar{D} \in \mathcal{K}$. Thus we have shown that $\widehat{\mathcal{K}} \subset \mathcal{K}$. The equality $\mathcal{K}=\widehat{\mathcal{K}}$ is proved.

From the definition of $\widehat{\mathcal{K}}$ (see (42)), it follows $\widehat{\mathcal{K}} \subset \overline{\mathcal{K}}$ and from Theorem 1 we get $\overline{\mathcal{K}} \subset \widehat{\mathcal{K}}$. These inclusions together with the equality $\mathcal{K}=\widehat{\mathcal{K}}$ imply $\mathcal{K}=\overline{\mathcal{K}}$.

Applying Corollary 1 with $Q=\mathcal{K}$, we conclude that there exists a matrix $\bar{D} \in \overline{\mathcal{K}}=\mathcal{K}$ such that relations (41) hold true. The lemma is proved.

It is worth noting that for the cone $\mathcal{K}$ defined in (35), the obtained in this section equivalent representations (37), (40), and (42) may be more preferable than the original definition, particularly (see the next section) when one needs to describe its dual cone.

\section{Alternative representations of the dual cone to the defined in (35) face of $\mathcal{C O P}^{p}$}

In this section, we will use the following statements proved in [25] (See Theorem 6.5 and Proposition 16.4.2)

Proposition 3 For two closed convex cones $C_{1}$ and $C_{2}$ in $\mathbb{R}^{m}$, it holds true

$$
\left(C_{1} \cap C_{2}\right)^{*}=\operatorname{cl}\left(C_{1}^{*} \oplus C_{2}^{*}\right) .
$$

If $\operatorname{relint}\left(C_{1}\right) \cap \operatorname{relint}\left(C_{2}\right) \neq \emptyset$, then

(i) $\left(C_{1}^{*} \oplus C_{2}^{*}\right)$ is a closed set and the closure operation in (45) can be omitted;

(ii) $\operatorname{relint}\left(C_{1} \cap C_{2}\right)=\operatorname{relint}\left(C_{1}\right) \cap \operatorname{relint}\left(C_{2}\right)$.

Here and in what follows, $C^{*}$ denotes the dual cone for a cone $C$ and $\oplus$ denotes the Minkowski sum.

Based on this proposition and definition (35), the dual cone to the cone $\mathcal{K}$ can be written in the form

$$
\mathcal{K}^{*}=\operatorname{cl} \mathcal{G}
$$

where

$$
\begin{gathered}
\mathcal{G}:=\left\{D \in \mathcal{S}(p): D=\sum_{i=1}^{p_{*}} \alpha_{i} \mu(i)(\mu(i))^{\top}+\sum_{i \in I}\left(\lambda(i)(t(i))^{\top}+t(i)(\lambda(i))^{\top}\right),\right. \\
\left.\mu(i) \in T, \alpha_{i} \geq 0, i=1, \ldots, p_{*} ; \lambda_{k}(i)=0 \forall k \in P \backslash L(i), \forall i \in I\right\},
\end{gathered}
$$

$p_{*}=p(p+1) / 2$. Let us give alternative descriptions of the dual cone $\mathcal{K}^{*}$. 
It follows from Lemma 3 that the cone $\mathcal{K}$ admits the following representation:

$$
\mathcal{K}=\mathcal{C O P}\left(Z_{\mathcal{K}}\right) \cap \mathcal{K}_{\text {pol }}
$$

where

$$
\begin{aligned}
\mathcal{C O P}\left(Z_{\mathcal{K}}\right):=\left\{D \in \mathcal{S}(p): t^{\top} D t \geq 0 \quad \forall t \in \Omega\left(Z_{\mathcal{K}}\right)\right\} \\
\mathcal{K}_{p o l}:=\left\{D \in \mathcal{S}(p): e_{k}^{\top} D \tau(j)=0 \quad \forall k \in M_{\mathcal{K}}(j),\right. \\
\left.e_{k}^{\top} D \tau(j) \geq 0 \forall k \in P \backslash M_{\mathcal{K}}(j), \forall j \in J_{\mathcal{K}}\right\},
\end{aligned}
$$

the sets $Z_{\mathcal{K}}=\left\{\tau(j), \in J_{\mathcal{K}}\right\}, M_{\mathcal{K}}(j), j \in J_{\mathcal{K}}$, and $\Omega\left(Z_{\mathcal{K}}\right)$ are defined in (4), (7) with $Q=\mathcal{K}$ and (10), (11) with $V=Z_{\mathcal{K}}$.

It is known (see [10]) that the cone $\mathcal{C O P}\left(Z_{\mathcal{K}}\right)$ is convex, closed, and pointed, its interior can be presented in the form

$$
\operatorname{int}\left(\mathcal{C O P}\left(Z_{\mathcal{K}}\right)\right)=\left\{D \in \mathcal{S}(p): t^{\top} D t>0 \forall t \in \Omega\left(Z_{\mathcal{K}}\right)\right\}
$$

and its dual cone is $\left(\mathcal{C O} \mathcal{P}\left(Z_{\mathcal{K}}\right)\right)^{*}=\operatorname{cl} \mathcal{G}\left(Z_{\mathcal{K}}\right)$, where

$$
\mathcal{G}\left(Z_{\mathcal{K}}\right):=\left\{D \in \mathcal{S}(p): D=\sum_{i=1}^{p_{*}} \alpha_{i} \mu(i)(\mu(i))^{\top}, \alpha_{i} \geq 0, \mu(i) \in \Omega\left(Z_{\mathcal{K}}\right) \forall i=1, \ldots, p_{*}\right\} .
$$

By construction, the set $\Omega\left(Z_{\mathcal{K}}\right)$ is closed and $\mathbf{e}^{\top} \mu=1, \mu \geq 0$ for any $\mu \in \Omega\left(Z_{\mathcal{K}}\right)$. Then we can show that $\left(\mathcal{C O P}\left(Z_{\mathcal{K}}\right)\right)^{*}=\mathcal{G}\left(Z_{\mathcal{K}}\right)$.

The cone $\mathcal{K}_{\text {pol }}$ is convex, closed, and for this cone it holds

relint $\mathcal{K}_{p o l} \supset\left\{D \in \mathcal{S}(p): e_{k}^{\top} D \tau(j)=0 \forall k \in M_{\mathcal{K}}(j) ; e_{k}^{\top} D \tau(j)>0 \forall k \in P \backslash M_{\mathcal{K}}(j), \forall j \in J_{\mathcal{K}}\right\}$,

$$
\begin{aligned}
&\left(\mathcal{K}_{p o l}\right)^{*}=\left\{D \in \mathcal{S}(p): D=\sum_{j \in J_{\mathcal{K}}}\left(\lambda(j)(\tau(j))^{\top}+\tau(j)(\lambda(j))^{\top}\right)\right. \\
&\left.\lambda_{k}(j) \geq 0 \forall k \in P \backslash M_{\mathcal{K}}(j), \forall j \in J_{\mathcal{K}}\right\} .
\end{aligned}
$$

Then it follows from Lemma 3 that there exists a matrix $\bar{D}$ such that

$$
\bar{D} \in \operatorname{int}\left(\mathcal{C O P}\left(Z_{\mathcal{K}}\right)\right) \cap \operatorname{relint} \mathcal{K}_{p o l}
$$

and based on Proposition 3 we conclude that

$$
\begin{aligned}
\operatorname{relint}(\mathcal{K}) & \left.\left.=\operatorname{relint}\left(\mathcal{C O} \mathcal{P}\left(Z_{\mathcal{K}}\right)\right) \cap \mathcal{K}_{\text {pol }}\right)=\operatorname{int}\left(\mathcal{C O P}\left(Z_{\mathcal{K}}\right)\right)\right) \cap \operatorname{relint}\left(\mathcal{K}_{\text {pol }}\right) \\
\mathcal{K}^{*} & =\left(\mathcal{C O P}\left(Z_{\mathcal{K}}\right) \cap \mathcal{K}_{\text {pol }}\right)^{*}=\left(\mathcal{C O P}\left(Z_{\mathcal{K}}\right)\right)^{*} \oplus\left(\mathcal{K}_{\text {pol }}\right)^{*}
\end{aligned}
$$

Thus we have proved the following theorem.

Theorem 3 Let the cone $\mathcal{K}$ be defined in (35). Then its dual cone can be described as $\mathcal{K}^{*}=\overline{\mathcal{G}}$, where

$$
\begin{aligned}
\overline{\mathcal{G}}:= & \left\{D \in \mathcal{S}(p): D=\sum_{i=1}^{p_{*}} \alpha_{i} \mu(i)(\mu(i))^{\top}+\sum_{j \in J_{\mathcal{K}}}\left(\lambda(j)(\tau(j))^{\top}+\tau(j)(\lambda(j))^{\top}\right),\right. \\
& \left.\alpha_{i} \geq 0, \mu(i) \in \Omega\left(Z_{\mathcal{K}}\right) \forall i=1, \ldots, p_{*} ; \lambda_{k}(j) \geq 0 \forall k \in P \backslash M_{\mathcal{K}}(j), \forall j \in J_{\mathcal{K}}\right\},
\end{aligned}
$$

and the sets $Z_{\mathcal{K}}=\left\{\tau(j), \in J_{\mathcal{K}}\right\}, M_{\mathcal{K}}(j), j \in J_{\mathcal{K}}$, and $\Omega\left(Z_{\mathcal{K}}\right)$ are defined in (4), (7) with $Q=\mathcal{K}$, and in (10), (11) with $V=Z_{\mathcal{K}}$. 
Notice that in the case when the information about the minimal zeros $\tau(j)$ and sets $M_{\mathcal{K}}(j), j \in J_{\mathcal{K}}$, is not available, one can use an alternative representation of the dual cone $\mathcal{K}^{*}$

$$
\mathcal{K}^{*}=\operatorname{cl} \widetilde{\mathcal{G}}
$$

where

$$
\begin{aligned}
\widetilde{\mathcal{G}}:=\left\{D \in \mathcal{S}(p): \quad D=\sum_{i=1}^{p_{*}} \alpha_{i} \mu(i)(\mu(i))^{\top}+\sum_{i \in I}\left(\lambda(i)(t(i))^{\top}+t(i)(\lambda(i))^{\top}\right),\right. \\
\left.\alpha_{i} \geq 0, \mu(i) \in T \forall i=1, \ldots, p_{*} ; \quad \lambda_{k}(i) \geq 0 \forall k \in P \backslash L(i), \forall i \in I\right\} .
\end{aligned}
$$

This description of the dual cone $\mathcal{K}^{*}$ is based on its representation (37) and formula (45).

The obtained here representations (49) and (50) of the dual cone $\mathcal{K}^{*}$ are preferable than formula (46) directly based on the definition (35) of the cone $\mathcal{K}$. This can be motivated by the following reasons:

- the inclusions $\mathcal{G} \subset \widetilde{\mathcal{G}} \subset \overline{\mathcal{G}}$ hold true and, in general, $\mathcal{G} \neq \widetilde{\mathcal{G}}$;

- to construct the set $\widetilde{\mathcal{G}}$, we need only the original data used in the definition (35) of the cone $\mathcal{K}$;

- in the representation $\mathcal{K}^{*}=\overline{\mathcal{G}}$, the closure operator is absent.

In fact, it is evident that $\mathcal{G} \subset \widetilde{\mathcal{G}}$.

Let us show that $\widetilde{\mathcal{G}} \subset \overline{\mathcal{G}}$. Suppose that $D \in \widetilde{\mathcal{G}}$, wherefrom by definition,

$$
D=\sum_{i \in I_{*}} \alpha_{i} \mu(i)(\mu(i))^{\top}+\sum_{i \in I}\left(\lambda(i)(t(i))^{\top}+t(i)(\lambda(i))^{\top}\right)
$$

where $\mu(i) \in T, \alpha_{i}>0 \quad \forall i \in I_{*} \subset\left\{1, \ldots, p_{*}\right\}, \lambda_{k}(i) \geq 0 \forall k \in P \backslash L(i), \forall i \in I$.

Since, by construction $t(i) \in \operatorname{conv} Z_{\mathcal{K}}, i \in I$, there exist numbers $\alpha_{i j} \geq 0, j \in J_{\mathcal{K}}$, $i \in I$, such that $t(i)=\sum_{j \in J_{\mathcal{K}}} \alpha_{i j} \tau(j) \forall i \in I$. Taking into account these equalities, we can rewrite equality (52) as follows:

$$
D=\sum_{i \in I_{*}} \alpha_{i} \mu(i)(\mu(i))^{\top}+\sum_{j \in J_{\mathcal{K}}}\left(\bar{\lambda}(j)(\tau(j))^{\top}+\tau(j)(\bar{\lambda}(j))^{\top}\right)
$$

where $\bar{\lambda}(j):=\sum_{i \in I} \alpha_{i j} \lambda(i), j \in J_{\mathcal{K}}$. Let us show that

$$
\bar{\lambda}_{k}(j) \geq 0 \quad \forall k \in P \backslash M_{\mathcal{K}}(j), \forall j \in J_{\mathcal{K}}
$$

Suppose that on the contrary, there exist $j_{0} \in J_{\mathcal{K}}$ and $k_{0} \in P \backslash M_{\mathcal{K}}\left(j_{0}\right)$, such that $\bar{\lambda}_{k_{0}}\left(j_{0}\right)=\sum_{i \in I} \alpha_{i j_{0}} \lambda_{k_{0}}(i)<0$. Hence, there exists $i_{0} \in I$ such that $\alpha_{i_{0} j_{0}}>0, \lambda_{k_{0}}\left(i_{0}\right)<0$. This implies $j_{0} \in J\left(i_{0}\right):=\left\{j \in J_{\mathcal{K}}: \alpha_{i_{0} j}>0\right\}, k_{0} \in L\left(i_{0}\right)$. From these relations and (44), it follows that $k_{0} \in M_{\mathcal{K}}\left(j_{0}\right)$. But this contradicts the condition $k_{0} \in P \backslash M_{\mathcal{K}}\left(j_{0}\right)$. Inequalities (54) are proved.

If $\mu(i) \in \Omega\left(Z_{\mathcal{K}}\right)$ for all $i \in I_{*}$, then from (53) and (54), it follows $D \in \overline{\mathcal{G}}$. Suppose, first, that there exists $i_{0} \in I_{*}$ such that $0<\rho\left(\mu\left(i_{0}\right)\right.$, conv $\left.Z_{\mathcal{K}}\right)<\sigma\left(Z_{\mathcal{K}}\right)$. Replacing $\mu\left(i_{0}\right)$ 
by $\bar{\mu}\left(i_{0}\right):=\beta \mu\left(i_{0}\right)$ and $\alpha_{i_{0}}$ by $\bar{\alpha}_{i_{0}}:=\alpha_{i_{0}} / \beta^{2}$ with $\beta:=\sigma\left(Z_{\mathcal{K}}\right) / \rho\left(\mu\left(i_{0}\right), \operatorname{conv} Z_{\mathcal{K}}\right)>0$, we obtain

$$
\alpha_{i_{0}} \mu(i)\left(\mu\left(i_{0}\right)\right)^{\top}=\bar{\alpha}_{i_{0}} \bar{\mu}\left(i_{0}\right)\left(\bar{\mu}\left(i_{0}\right)\right)^{\top}, \bar{\mu}\left(i_{0}\right) \in \Omega\left(Z_{\mathcal{K}}\right) .
$$

Now suppose that there exists $i_{0} \in I_{*}$ such that $\rho\left(\mu\left(i_{0}\right)\right.$, conv $\left.Z_{\mathcal{K}}\right)=0$. Hence

$$
\mu\left(i_{0}\right)=\sum_{j \in \bar{J}} \beta_{j} \tau(j), \beta_{j}>0 \quad \forall j \in \bar{J} \subset J_{\mathcal{K}}
$$

From these relations we conclude that

$$
\begin{aligned}
& P_{0}\left(\mu\left(i_{0}\right)\right) \subset P_{0}(\tau(j)) \quad \forall j \in \bar{J}, \\
& \alpha_{i_{0}} \mu\left(i_{0}\right)\left(\mu\left(i_{0}\right)\right)^{\top}=\frac{\alpha_{i_{0}}}{2} \sum_{j \in \bar{J}} \beta_{j}\left(\tau(j)\left(\mu\left(i_{0}\right)\right)^{\top}+\mu\left(i_{0}\right)(\tau(j))^{\top}\right) .
\end{aligned}
$$

Set $\bar{\alpha}_{i_{0}}=0, \tilde{\lambda}(j)=\bar{\lambda}(j)+\alpha_{i_{0}} \beta_{j} \mu\left(i_{0}\right), j \in \bar{J}$, and $\tilde{\lambda}(j)=\bar{\lambda}(j), i \in J_{\mathcal{K}} \backslash \bar{J}$.

From (53)-(56), it follows that the matrix $D \in \widetilde{\mathcal{G}}$ can be written in the form (49) with $\alpha_{i}, \mu(i), i \in I_{*}$, and $\lambda(j), j \in J_{\mathcal{K}}$, replaced by $\bar{\alpha}_{i}, \bar{\mu}(i), i \in I_{*} ; \tilde{\lambda}(j), j \in J_{\mathcal{K}}$, such that $\tilde{\lambda}_{k}(j) \geq 0 \quad \forall k \in P \backslash M_{\mathcal{K}}(j), \forall j \in J_{\mathcal{K}}$, and $\bar{\alpha}_{i}>0 \Rightarrow \bar{\mu}(i) \in \Omega\left(Z_{\mathcal{K}}\right) \forall i \in I_{*}$. Therefore, we conclude that $D \in \overline{\mathcal{G}}$ and, hence, $\widetilde{\mathcal{G}} \subset \overline{\mathcal{G}}$.

To show that, in general, $\mathcal{G} \neq \widetilde{\mathcal{G}}$, let us consider an example.

Let $p=2, I=\{1\}, t(1)=(1,0)^{\top}, L(i)=\{1\}, \lambda(1)=(0,1)^{\top}$. Then

$$
D_{*}=\lambda(1)(t(1))^{\top}+t(1)(\lambda(1))^{\top}=\left(\begin{array}{cc}
0 & 1 \\
1 & 0
\end{array}\right) \in \widetilde{\mathcal{G}} .
$$

It is easy to see that the matrix $D_{*}$ cannot be presented in the form

$$
D_{*}=\sum_{i=1}^{3} \alpha_{i} \mu(i)(\mu(i))^{\top}+\bar{\lambda}(1)(t(1))^{\top}+t(1)(\bar{\lambda}(1))^{\top},
$$

where $\mu(i) \in T, \alpha_{i} \geq 0, i=1,2,3 ; \bar{\lambda}(1)=\left(\bar{\lambda}_{1}(1), 0\right)$. Hence $D_{*} \notin \mathcal{G}$.

\section{The minimal face of $\mathcal{C O} \mathcal{P}^{p}$ containing a given con- vex set}

Let $Q$ be a convex closed subset of $\mathcal{C O} \mathcal{P}^{p}$ with the corresponding sets $T_{0}(Q)$ and $Z_{Q}=$ $\left\{\tau(j), j \in J_{Q}\right\}$ of all zeros and minimal zeros of $Q$ defined in (2) and (4), and the sets $M_{Q}(j), j \in J_{Q}$, defined by relations $(7)$.

Consider the cone

$$
\mathcal{K}_{Q}:=\left\{D \in \mathcal{C O O} \mathcal{P}^{p}: e_{k}^{\top} D \tau(j)=0 \forall k \in M_{Q}(j), \forall j \in J_{Q}\right\}
$$

It is evident that $Q \subset \mathcal{K}_{Q}$ and it is easy to show that

$$
T_{0}\left(\mathcal{K}_{Q}\right)=T_{0}(Q), Z_{\mathcal{K}_{Q}}=Z_{Q}, M_{\mathcal{K}_{Q}}(j)=M_{Q}(j) \forall j \in J_{Q}=J_{\mathcal{K}_{Q}}
$$


Then from the results of sections 3 and 4 , one can conclude that $\mathcal{K}_{Q}$ is a face of $\mathcal{C} \mathcal{O P}^{p}$ and

$$
\operatorname{relint}\left(\mathcal{K}_{Q}\right)=\operatorname{relint}\left(\mathcal{C O P}\left(Z_{Q}\right) \cap \mathcal{K}_{\text {pol }}^{Q}\right)=\operatorname{relint}\left(\mathcal{C O P}\left(Z_{Q}\right)\right) \cap \operatorname{relint}\left(\mathcal{K}_{\text {pol }}^{Q}\right) \text {, }
$$

where the sets $\Omega\left(Z_{Q}\right)$ and $\mathcal{C O P}\left(Z_{Q}\right)$ are defined by the rules (10), (11), and (48) using the minimal zeros set $Z_{Q}$ and

$$
\mathcal{K}_{\text {pol }}^{Q}:=\left\{D \in \mathcal{S}(p): e_{k}^{\top} D \tau(j)=0 \forall k \in M_{Q}(j) ; e_{k}^{\top} D \tau(j) \geq 0 \forall k \in P \backslash M_{Q}(j), j \in J_{Q}\right\} .
$$

It follows from (34) and (57) that there exists a matrix $\bar{D}$ such that

$$
\bar{D} \in \operatorname{relint}\left(\mathcal{K}_{Q}\right) \cap Q \neq \emptyset \text {. }
$$

The following Proposition is proved in [22] (see Proposition 3.2.2).

Proposition 4 Let $F$ be a face of a convex cone $K$ and $Q$ a convex subset of $K$. If $Q \subset F$ and $Q \cap \operatorname{relint} F \neq \emptyset$ then $F=\operatorname{face}(Q, K)$.

Here and in what follows face $(S, C)$ denotes the minimal (by inclusion) face of a cone $C$ containing a set $S$.

Theorem 4 Let $Q$ be a convex closed subset of $\mathcal{C O P}^{p}$. Then

$$
\operatorname{face}\left(Q, \mathcal{C O} \mathcal{P}^{p}\right)=\mathcal{K}_{Q}:=\left\{D \in \mathcal{C O} \mathcal{P}^{p}: e_{k}^{\top} D \tau(j)=0 \forall k \in M_{Q}(j), \forall j \in J_{Q}\right\},
$$

where $\left\{\tau(j), j \in J_{Q}\right\}$ is the set of all minimal zeros of $Q$ and the sets $M_{Q}(j), j \in J_{Q}$, are defined in (7).

Proof. The statement of this theorem follows from condition (58) and Proposition 4.

Corollary 2 Any face of $\mathcal{C O P}^{p}$ can be presented in the form (35) with some vectors $t(i) \in T$ and sets $L(i), P_{+}(t(i)) \subset L(i) \subset P, \quad \forall i \in I, 0 \leq|I|<\infty$.

Proof. Let $F$ be a face of the cone $\mathcal{C O}^{p}$. It is known that $F$ is a convex closed subset of $\mathcal{C O} \mathcal{P}^{p}$. Applying Theorem 4 with $Q=F$, we obtain

$$
\operatorname{face}\left(F, \mathcal{C O} \mathcal{P}^{p}\right)=\left\{D \in \mathcal{C O} \mathcal{P}^{p}: e_{k}^{\top} D \tau(j)=0 \forall k \in M_{F}(j), \forall j \in J_{F}\right\},
$$

where $\left\{\tau(j), j \in J_{F}\right\}$ is the set of all minimal zeros of $F$ and the sets $M_{F}(j), j \in J_{F}$, are defined in (7) with $Q=F$. Taking into account the evident equality face $\left(F, \mathcal{C O} \mathcal{P}^{p}\right)=F$, we conclude that $F$ can be represented in the form (35), namely

$$
F=\left\{D \in \mathcal{C O}^{p}: e_{k}^{\top} D \tau(j)=0 \forall k \in M_{F}(j), \forall j \in J_{F}\right\} .
$$

The corollary is proved.

Consider a matrix $A \in \mathcal{C O P}^{p}$ and the corresponding set of all its minimal zeros $Z_{A}=\left\{\tau(j), j \in J_{A}\right\}$.

Corollary 3 The minimal face of $\mathcal{C O}^{p}$ containing a given copositive matrix $A$ is as follows:

$$
\operatorname{face}\left(A, \mathcal{C O} \mathcal{P}^{p}\right)=\left\{D \in \mathcal{C O} \mathcal{P}^{p}: e_{k}^{\top} D \tau(j)=0 \forall k \in M_{A}(j), \forall j \in J_{A}\right\},
$$

where $M_{A}(j)=\left\{k \in P: e_{k}^{\top} A \tau(j)=0\right\}, j \in J_{A}$.

Notice that this corollary correlates with results from [9], where for a given matrix $A \in \mathcal{C O} \mathcal{P}^{p}$, the author gives explicit description for $\operatorname{span}\left\{\operatorname{face}\left(A, \mathcal{C O} \mathcal{P}^{p}\right)\right\}$. 


\section{On equivalent descriptions of the feasible set of a copositive problem}

Consider a convex copositive problem in the form

$$
\min _{x \in \mathbb{R}^{n}} c(x) \text { s.t. } \mathcal{A}(x) \in \mathcal{C} \mathcal{O} \mathcal{P}^{p},
$$

where $x=\left(x_{1}, \ldots, x_{n}\right)^{\top}$ is the vector of decision variables, $c: \mathbb{R}^{n} \rightarrow \mathbb{R}$ is a convex function, and $\mathcal{A}: \mathbb{R}^{n} \rightarrow \mathcal{S}(p)$ is a given matrix function such that for any $x \in \mathbb{R}^{n}, y \in \mathbb{R}^{n}$, the following inclusions are satisfied:

$$
\mathcal{A}(\lambda x+(1-\lambda) y)-\lambda \mathcal{A}(x)-(1-\lambda) \mathcal{A}(y) \in \mathcal{C O} \mathcal{P}^{p} \forall \lambda \in[0,1] .
$$

The aim of this section is to prove Theorem 5 (see below) and use it to obtain equivalent useful descriptions of the feasible set of problem (59). This set can be written in the form

$$
X:=\left\{x \in \mathbb{R}^{n}: \mathcal{A}(x) \in \mathcal{C O} \mathcal{P}^{p}\right\}=\left\{x \in \mathbb{R}^{n}: t^{\top} \mathcal{A}(x) t \geq 0 \quad \forall t \in T\right\},
$$

where $\mathcal{A}(x)$ is a matrix function satisfying (60) and the set $T$ is defined in (1).

Let $\mathcal{D}$ be a subset of the cone $\mathcal{C O} \mathcal{P}^{p}$ given as follows:

$$
\mathcal{D}=\{D: D=\mathcal{A}(x), x \in X\} .
$$

Evidently, the set $\mathcal{D}$ is closed, convex, and the corresponding set of all its zeros (see (2)) can be written in the form

$$
T_{0}(\mathcal{D})=\left\{t \in T: t^{\top} \mathcal{A}(x) t=0 \forall x \in X\right\} .
$$

Suppose that the set $T_{0}(\mathcal{D})$ is not empty. Consider a finite non-empty subset of this set

$$
\mathcal{V}:=\left\{t(i) \in T_{0}(\mathcal{D}), i \in \mathcal{I}\right\}, 0<|\mathcal{I}|<\infty .
$$

The following theorem permits us to characterize the set (61) in terms of zeros of the corresponding set $\mathcal{D}$.

Theorem 5 Given the closed convex set $X$ defined in (61), the corresponding subset $\mathcal{D}$ of the cone $\mathcal{C O P}^{p}$ defined in (62), and any subset (64) of the set $T_{0}(\mathcal{D})$ defined in (63), the following equality holds: $X=\mathcal{X}(\mathcal{V})$, where

$$
\mathcal{X}(\mathcal{V}):=\left\{x \in \mathbb{R}^{n}: \mathcal{A}(x) t(i) \geq 0 \forall i \in \mathcal{I} ; \quad t^{\top} \mathcal{A}(x) t \geq 0 \forall t \in \Omega(\mathcal{V})\right\},
$$

and the set $\Omega(\mathcal{V})$ is constructed by the rules (9)-(11) with $V=\mathcal{V}$.

Proof. Since, by construction, $t(i) \in T_{0}(\mathcal{D}) \forall i \in \mathcal{I}$, it is easy to show that

$$
e_{k}^{\top} \mathcal{A}(x) t(i)=0 \forall k \in P_{+}(t(i)) ; e_{k}^{\top} \mathcal{A}(x) t(i) \geq 0 \forall k \in P_{0}(t(i)), \forall i \in \mathcal{I}, \forall x \in X .
$$

It follows from these relations that $X \subset \mathcal{X}(\mathcal{V})$.

Now, consider any $x \in \mathcal{X}(\mathcal{V})$. By construction,

$$
\mathcal{A}(x) t(i) \geq 0 \forall i \in \mathcal{I} ; \quad t^{\top} \mathcal{A}(x) t \geq 0 \forall t \in \Omega(\mathcal{V}) .
$$

It follows from Theorem 1 that the relations above imply the inequalities $t^{\top} \mathcal{A}(x) t \geq 0$ for all $t \in T$ and, consequently, $x \in X$. Hence, we have shown that $\mathcal{X}(\mathcal{V}) \subset X$. The theorem is proved. 
Remark 3 Theorem 5 can be considered as a generalization of Lemma 2 from [16], where it was proved that for the set $X$ defined in (61), there exists a number $\sigma_{*}>0$ such that

$$
\begin{aligned}
X=\left\{x \in \mathbb{R}^{n}\right. & : \mathcal{A}(x) t(i) \geq 0 \forall i \in \mathcal{I} \\
& t^{\top} \mathcal{A}(x) t \geq 0 \forall t \in\left\{t \in T: \rho(t, \operatorname{conv}\{t(i), i \in \mathcal{I}\}) \geq \sigma_{*}\right\},
\end{aligned}
$$

but no rules have been given to find this number. Theorem 5 states that one can easily compute $\sigma_{*}$ by the formula $\sigma_{*}=\sigma(\mathcal{V})=\min \left\{t_{k}(i), k \in P_{+}(t(i)), i \in \mathcal{I}\right\}$, where $\mathcal{V}$ is defined in (64).

Remark 4 It is easy to see that all the results of this section remain fair if

- replace the condition $x \in \mathbb{R}^{n}$ by the condition $x \in X_{*}$ with a convex closed set $X_{*} \subset \mathbb{R}^{n}$,

- given a set of indices $\mathcal{V}$ in the form (64), replace the number $\sigma(\mathcal{V})$ defined in (10) by the number $\sigma_{*}(\mathcal{V}):=\min \left\{t_{k}(i), k \in P_{+}(t(i)), i \in \mathcal{I}_{*}\right\} \geq \sigma(\mathcal{V})$, where $\left\{t(i), i \in \mathcal{I}_{*}\right\}$, $\mathcal{I}_{*} \subset \mathcal{I}$, is the set of vertices of the set conv $\mathcal{V}$.

The statement of Theorem 5 is useful for study of linear copositive problems since now we can obtain "good" description of the set $X$ and regularize this problem.

In fact, suppose that in problem (59), the matrix function $\mathcal{A}(x)$ is linear and its constraints do not satisfy the Slater condition. Consider the defined in $(62)$ set $\mathcal{D}$. Let $Z_{\mathcal{D}}:=\left\{\tau(j), j \in J_{\mathcal{D}}\right\}$ be the set of all minimal zeros of $\mathcal{D}$. It follows from Lemma 1 that $1 \leq\left|J_{\mathcal{D}}\right|<\infty$.

Consider the SIP problem

$$
\min c(x) \text {, s.t. } \mathcal{A}(x) \tau(j) \geq 0 \forall j \in J_{\mathcal{D}}, t^{\top} \mathcal{A}(x) t \geq 0 \forall t \in \Omega\left(Z_{\mathcal{D}}\right) .
$$

This problem can be considered as a regularization of the original linear CoP problem (59) since it is equivalent to this problem (see Theorem 5), has a finite number of linear inequality constraints $\mathcal{A}(x) \tau(j) \geq 0, j \in J_{\mathcal{D}}$, and there exists $\bar{x} \in X$ such that $t^{\top} \mathcal{A}(\bar{x}) t>$ $0 \forall t \in \Omega\left(Z_{\mathcal{D}}\right)$ (see Theorem 2 in section 3 or Lemma 3 in [16]).

Let $\mathbf{F}_{\min }$ be the smallest (by inclusion) face of $\mathcal{C O} \mathcal{P}^{p}$ containing the set $\mathcal{D}$ defined by formula (62) in terms of the constraints of problem (59). The face $\mathbf{F}_{\min }$ is called (see [26]) the minimal face of this optimization problem.

For the copositive problem (59), we can formulate the following corollary from Theorem 4 proved in the previous section.

Corollary 4 The minimal face of problem (59) has the form

$$
\mathbf{F}_{\text {min }}=\left\{D \in \mathcal{C O} \mathcal{P}^{p}: e_{k}^{\top} D \tau(j)=0 \forall k \in M_{\mathcal{D}}(j), \forall j \in J_{\mathcal{D}}\right\},
$$

where the set $\mathcal{D}$ is defined in (62), $\left\{\tau(j), j \in J_{\mathcal{D}}\right\}$ is the set of all minimal zeros of $\mathcal{D}$, and the sets $M_{\mathcal{D}}(j), j \in J_{\mathcal{D}}$, are defined by formulas (7) with $Q$ substituted by $\mathcal{D}$ :

$$
M_{\mathcal{D}}(j):=\left\{k \in P: e_{k}^{\top} \mathcal{A}(x) \tau(j)=0 \forall x \in X\right\}, j \in J_{\mathcal{D}} .
$$

The importance of Corollary 4 lies in the fact that it describes the way of representing the minimal face of the copositive problem (59) in an explicit form using the minimal zeros of the set $\mathcal{D}$. This representation may be useful for creating new numerical methods based on the minimal cone representations. 


\section{Conclusions}

The results of the paper permit to explicitly describe faces of the cone of copositive matrices and the respective dual cones in terms of the minimal zeros of these faces. The novelty of the obtained results consists of the fact that they give a new perception of the facial structure of the cone $\mathcal{C O P}^{p}$ which is not well investigated yet [4]. In his paper [21] dedicated to the characterization of more simple cases of convex cones (so-called nice cones), Gabor Pataki wrote: "...Copositive, and completely positive cones lie at the other end of the spectrum. Though they are very useful in optimization ..., optimizing over them is more difficult. Also, while considerable progress has been made in describing their geometry ..., a complete understanding (such as a complete description of their facial structure) is probably out of reach."

We expect that the results of this paper will help to better understand the facial structure of the cone $\mathcal{C O} \mathcal{P}^{p}$ and describe its faces explicitly.

Moreover, the obtained in the paper representations are useful for the study of convex copositive problems. In particular, they permit to

- create regularization procedures based on the face reduction approach;

- formulate for CoP problems new optimality conditions without any CQs;

- develop new strong duality theory for copositive optimization based on an explicit formulation of the Extended Lagrange Dual Problem;

- develop numerical methods for solving CoP problems.

\section{Acknowledgement}

This work was supported by the state research program "Convergence" (Republic Belarus), Task 1.3.01, Portuguese funds through CIDMA - Center for Research and Development in Mathematics and Applications, and FCT - Portuguese Foundation for Science and Technology, within the project UIDB/04106/2020.

\section{References}

[1] Ahmed F, Dür M, Still G. Copositive Programming via semi-infinite optimization. J. Optim. Theory Appl. 2013;159:322-340.

[2] Anjos MF, Lasserre JB, editors. Handbook of Semidefinite, Conic and Polynomial Optimization. Springer US; 2012. International Series in Operational Research and Management Science; vol. 166.

[3] Afonin A, Hildebrand R, Dickinson P. The extreme rays of the $6 \times 6$ copositive cone. J Glob Optim. 2020. Available from: https://doi.org/10.1007/s10898-020-00930-y.

[4] Berman A, Dür M, Shaked-Monderer N. Open problems in the theory of completely positive and copositive matrices. Electron J Linear Algebra. 2015; 29:46-58. 
[5] Bomze IM. Copositive optimization - recent developments and applications. EJOR 2012; 216(3): 509-520.

[6] Dür M. Copositive Programming - a Survey. In: Diehl M, Glineur F, Jarlebring E, Michielis W., editors. Recent advances in optimization and its applications in engineering; 2010; Springer-Verlag, Berlin, Heidelberg: 535 p.

[7] Drusvyatskiy D, Wolkowicz H. The many faces of degeneracy in conic optimization. Foundations and Trends in Optimization, Now Publishers Inc.; 2017; 3(2): 77-170.

[8] Dickinson PJC. Geometry of the copositive and completely positive cones, J Math Anal Appl. 2011; 380: 377 - 395.

[9] Dickinson PJC, Hildebrand R. Considering copositivity locally, J Math Anal Appl. 2016; 437(2): 1184-1195.

[10] Eichfelder G, Povh J. On the set-semidefinite representation of nonconvex quadratic programs over arbitrary feasible sets. Optim Lett. 2013; 7: 1373-1386.

[11] Hildebrand R. The extreme rays of the $5 \times 5$ copositive cone. Linear Algebra its Appl. 2012; 437(7): 1538-1547.

[12] Jongen HT, Twilt F, Weber G.-W. Semi-infinite optimization: structure and stability of the feasible set. J. Optim. Theory Appl. 1992; 72: 529 - 552.

[13] Klatte D, Henrion R. Regularity and stability in nonlinear semi-infinite optimization. In: Reemstsen R, Ruckmann JJ., editors. Semi-Infinite Programming: 1998; Kluwer, Dordrecht. p. 69 - 102.

[14] Hettich R, Kortanek KO. Semi-infinite programming: theory, methods, and applications. SIAM Rev. 1993; 35: 380 - 429.

[15] Kostyukova OI, Tchemisova TV. Optimality conditions for convex Semi-Infinite Programming problems with finitely representable compact index sets. J Optim Theory Appl. 2017; 175(1): 76-103.

[16] Kostyukova OI, Tchemisova TV, Dudina OS. Immobile indices and CQ-free optimality criteria for linear copositive programming problems. Set-Valued Var Anal. 2020; 28: 89-107.

[17] Kostyukova OI, Tchemisova TV. CQ-free optimality conditions and strong dual formulations for a special conic optimization problem. SOIC. 2020; 3(3): 668 - 683.

[18] Liu YL, Pan SH. Strong calmness of perturbed KKT system for a class of conic programming with degenerate solutions. Optimization. 2019; 68(6):1131-1156.

[19] Lopez M, Still G. Semi-infinite programming. Eur J Oper Res. 2007; 180(2): 491-518.

[20] Mordukhovich B, Nghia TTA. Constraint qualifications and optimality conditions for nonconvex semi-infinite and infinite programs. Math. Program., Ser. B. 2013; 139: $271-300$. 
[21] Pataki G. On the connection of facially exposed and nice cones. J Math Anal Appl. 2013; 400(1): 211-221.

[22] Pataki G. The geometry of semidefinite programming. In: Wolkowicz H, Saigal R, Vandenberghe L., editors. Handbook of semidefinite programming. 2000; Kluwers Academic, Amsterdam; p. 29-65.

[23] Ramana MV. An exact duality theory for Semidefinite Programming and its complexity implications. Math. Prog. 1997; 77:129-162.

[24] Ramana MV, Tuncel L, Wolkowicz H. Strong duality for Semidefinite Programming. SIAM J. Optimization.1997; 7(3):641-662.

[25] Rockafellar RT. Convex analysis. Princeton university press. 1970.

[26] Waki H, Muramatsu M. Facial reduction algorithms for conic optimization problems, J Optim Theory Appl. 2013; 158:188-215.

[27] Weber GW, Kropat E, Alparslan Gök SZ. Semi-Infinite and conic optimization in modern human life and financial sciences under uncertainty. In: ISI Proceedings of 20th Mini-EURO conference; 2008. p. 180-185.

[28] Wolkowicz H, Saigal R, Vandenberghe L. Handbook of Semidefinite Programming theory, algorithms, and applications. Kluwer Academic Publishers. 2000. 